\title{
Incidental recognition memory for concrete and abstract sentences equated for comprehensibility
}

\author{
NICHOLAS A. KUIPER \\ University of Calgary, Calgary, Alberta, Canada T2N $1 N_{4}$ \\ and \\ ALLAN PAIVIO \\ University of Western Ontario, London, Ontario, Canada N6A SK7
}

\begin{abstract}
Twenty-four subjects rated a set of concrete and abstract sentences on comprehensibility. They were then given an unexpected memory test, involving recognition confidence ratings of a set of sentences (which included the same ones they had originally rated on comprehensibility), synonyms of those sentences, and new unrelated distractors. The results showed that, with comprehensibility equated, concrete sentences exceeded abstract in terms of confidence ratings for correct acceptance of same and correct rejection of new sentences. In addition, as predicted from a dual coding theory, concrete synonyms received higher false positive confidence ratings than did abstract synonyms. Dual coding and common code theories were discussed in the light of these findings.
\end{abstract}

Begg and Paivio (1969) found that subjects exposed to concrete sentences detected subsequent semantic changes (subject-object reversals) better than lexical (synonym) changes, while the results for abstract sentences were reversed. These findings were explained in terms of a dual coding hypothesis, in which the meaning of concrete material was presumed to be stored primarily in the imaginal system and abstract material primarily in the verbal system.

Johnson, Bransford, Nyberg, and Cleary (1972) suggested that the interaction found by Begg and Paivio (1969) may have resulted partly from initial differences in comprehension, with abstract sentences being more difficult to comprehend than concrete sentences. Subsequent studies have attempted to remove this source of confounding by equating concrete and abstract sentences, using independent ratings on a 7-point "ease of understanding" scale (Moeser, 1974; Rowe, Schurr, and Meisinger, Note 1). Using this procedure, Rowe et al. (Note 1) found that cued recall of sentence content was much greater for concrete sentences $(37 \%)$ than for abstract sentences (9\%), with no effect of cuing (grammatical subject vs grammatical object) or syntactic form of the sentence (active, passive, and interrogative) upon recall. Moeser (1974), in a series of experiments, also presented subjects with concrete and abstract sentences that had been equated on comprehensibility. Contrary to the Begg and Paivio

Requests for reprints should be sent to Nicholas A. Kuiper, Department of Psychology, University of Calgary, Calgary, Alberta, Canada T2N 1N4.
(1969) finding, Moeser found that, in nearly all instances, subjects were better at identifying both semantic and lexical changes in concrete sentences than in abstract sentences.

The present study examined the use of such a rating procedure to equate the comprehensibility of concrete and abstract verbal material as well as to induce different modes of encoding prior to an incidental memory test. Dual coding theory suggests that the comprehension of abstract and concrete sentences may involve imaginal and verbal mechanisms to different degrees (Paivio \& Begg, 1971). Abstract sentences may be comprehended primarily on the basis of representations of the individual words and intraverbal associations, while concrete sentences may be comprehended primarily on the basis of a holistic idea, represented by an image. Therefore, "equivalent" comprehension ratings may represent differential processing of concrete and abstract sentences, with different consequences on a memory test.

The present study attempted to test the above idea by having experimental subjects rate the comprehensibility of concrete and abstract sentences that had been equated on ease of comprehension according to ratings by an independent group. The experimental group was given a surprise recognition confidence rating task, in which some of the sentences had lexical changes that preserved meaning (synonyms), some were unchanged (sames), and some were new sentences (news). Several predictions were made on the basis of dual coding theory. Most notably, it was predicted that concrete synonym sentences would obtain higher false positive recognition ratings than would abstract 
synonyms, inasmuch as the imaginal system is hypothesized to preserve meaning but not specific wording of concrete material.

In addition, abstract same and synonym sentences were expected to differ such that the synonyms would have a lower positive recognition confidence rating. This prediction reflects the hypothesized verbal processing and storage of abstract material, which should favor detection of the lexical change across the samesynonym conditions of the present study. Conversely, concrete same and synonym ratings were not expected to differ because their meanings (as processed during the comprehension rating task) would be represented in terms of imagery, which should remain constant across the same-synonym conditions.

\section{METHOD}

\section{Subjects}

Forty-six students from introductory psychology courses at the University of Western Ontario served as subjects in partial fulfillment of course requirements. Twenty-four subjects were used in the main study, while two groups of 11 subjects each were used to obtain comprehension and synonymity ratings for the concrete and abstract sentences prior to the main study.

\section{Sentence Construction and Selection}

Sixty-eight pairs of abstract sentences and 68 pairs of concrete sentences were constructed using the frame, "The (adjective) (noun) (past-tense verb) a(n)/the (adjective) (noun)." Examples of the sentence pairs generated are presented in Table 1 .

A 7-point "ease of understanding" scale, similar to that employed by Rowe et al. (Note 1) was used to obtain comprehension ratings for individual concrete and abstract sentences. Synonymity ratings for each pair of sentences were obtained on a 7-point scale, where 1 indicated "low similarity of meaning" and 7 indicated "high similarity of meaning." The nine pairs of concrete and nine pairs of abstract sentences selected for the experiment were matched on both comprehension and synonymity ratings. A t test $[t(10)=.71$, n.s.] showed that the average comprehension rating of 5.24 for the 18 abstract sentences was not significantly different from the 5.86 average rating for the 18 concrete sentences. The average synonymity rating for the nine pairs of concrete sentences was 5.89 , with a $t$ test $[t(10)=1.74$, n.s.] showing this to be not significantly different from the abstract sentence pairs' average synonymity rating of 5.71. The nouns of the 18 concrete and 18 abstract sentences were checked against the imagery ratings of Paivio, Yuille, and Madigan (1968). The imagery ratings of concrete

Table 1

Examples of Concrete and Abstract Sentence Pairs

\section{Concrete}

A The colorful snake crushed a screaming boy.

B The colorful serpent crushed a screaming lad.

A The friendly magistrate purchased a green boat.

B The friendly judge purchased a green ship.

\section{Abstract}

A The latest evidence suggested a different option.

B The latest proof suggested a different alternative.

A The annual statement recommended an increased watchfulness.

B The annual report recommended an increased vigilance. nouns included in Paivio et al. (1968) averaged 6.42, while the abstract nouns included averaged 3.05.

\section{Design}

A 2 by 3 factorial design with repeated measures on both factors was used. The first factor was concreteness (concrete, abstract) and the second factor was sentence type (same, synonym, new).

\section{Material Presentation and Procedure}

All sentences were typed individually in uppercase on $19.20 \times 8.96 \mathrm{~cm}$ cards. The experiment was conducted in two parts. Subjects, tested in groups of one to six, were exposed to the concrete and abstract sentences by being asked to give comprehension ratings. Ratings were made on each card by using a 7-point scale where 1 was "very difficult to understand" and 7 was "very easy to understand." Upon completion of this task, subjects were given a second deck of cards containing concrete and abstract same, synonym, and new sentences. The subjects' task was to give a recognition confidence rating for each sentence in the deck, using the range of numbers from 1 to 7 , where 1 indicated that the subject was very sure the sentence had not been previously presented and 7 indicated that the subject was very sure the sentence had been presented earlier. Prior to the task, subjects were informed that some of the sentences were new, but they were not told of the meaningpreserving wording changes in some of the sentences.

The 18 concrete and 18 abstract sentences were completely counterbalanced across blocks of six subjects to insure that both the $A$ and $B$ members of each concrete and abstract sentence pair appeared in each condition (same, synonym, and new) and that every sentence appeared in all conditions. Both the comprehension and recognition confidence rating decks contained filler sentences from the pool of concrete and abstract sentences initially generated. The comprehension rating decks each had a total of 17 concrete and 17 abstract sentence fillers. Seventeen of the fillers (mixed concrete and abstract) preceded the six concrete and six abstract target sentences, while 17 fillers followed. Subject to this limitation, sentences were presented in different orders for each of the 24 comprehension decks.

The recognition confidence rating decks each contained a total of 38 sentences. The nine concrete and nine abstract target sentences were preceded by 20 fillers (five concrete and five abstract olds, five concrete and five abstract news). Again, subject to this limitation, sentences were presented in different orders for each of the 24 decks.

\section{RESULTS}

The mean recognition confidence ratings for abstract and concrete sentences as a function of sentence type (same, synonym, new) are presented in Table 2. A two-way analysis of variance, with concreteness (concrete, abstract) and sentence type (same, synonym, new) as the independent factors, was performed on the data, yielding significant effects of concreteness $[F(1,23)=13.34, \quad p<.01]$ and sentence type $[\mathrm{F}(2,46)=105.13, \mathrm{p}<.01]$, and an interaction between the two $[F(2,46)=30.98, p<.01]$. A min $F^{\prime}$ value was also calculated for the Concreteness by Sentence Type interaction, using the formula described by Clark $(1973 a)$, yielding a significant $\min F^{\prime}(2,109)=15.76$ $(\mathrm{p}<.001)$.

A Newman-Keuls analysis of the mean recognition confidence ratings presented in Table 2 revealed that all possible comparisons, except the concrete-same vs 
Table 2

Mean Recognition Confidence Ratings for Concrete and Abstract Sentences as a Function of Sentence Type

\begin{tabular}{cccc}
\hline & \multicolumn{3}{c}{ Sentence Type } \\
\cline { 2 - 4 } Concreteness & Same & Synonym & New \\
\hline Concrete & 6.40 & 5.92 & 1.30 \\
Abstract & 5.09 & 3.62 & 2.37 \\
\hline
\end{tabular}

concrete-synonym comparison, were significantly different. For the same condition, concrete sentences had a higher true positive recognition rating than did abstract sentences $(p<.01)$. In the synonym condition, the concrete sentence rating was again numerically higher than the abstract, indicating in this case that the concrete sentences received higher false positive recognition ratings $(p<.01)$. These positive ratings were considered false, as the subjects had not seen the specific sentences in the prior rating task. Regarding the new condition, concrete sentences received significantly lower ratings than abstracts $(p<.05)$, suggesting subjects were better at rating concrete sentences as true negatives. The Newman-Keuls analysis also revealed that, as predicted, abstract synonym ratings were significantly lower than abstract same ratings $(\mathrm{p}<.01)$.

The comprehension ratings obtained from the experimental group showed that concrete sentences exhibited a slightly higher comprehension rating than did abstract sentences $\quad[6.05$ vs 5.74, $t(23)=2.06, \quad p<.05]$. Accordingly, the data were analyzed post hoc to establish the contribution of the difference in concrete and abstract comprehension ratings to the pattern of recognition confidence ratings. A subset of 12 concrete and 12 abstract sentences were matched on comprehension ratings by the experimental group, yielding means of 6.25 and 6.21 , respectively, with equivalent standard deviations (.46 and .42 ). Average recognition confidence ratings were calculated for the same, synonym, and new conditions for only this subset of selected sentences. All of the ratings fell within the appropriate $95 \%$ confidence intervals calculated for each of the mean ratings reported in Table 2 , suggesting that comprehension differences had a minimal effect on the recognition confidence ratings obtained.

\section{DISCUSSION}

The results of the present study supported a dual coding interpretation, even when the set of concrete and abstract sentences were equated on comprehensibility. Concrete sentences with lexical word changes that preserved meaning (synonym condition) had higher false positive recognition ratings than did abstract sentences that had been similarly changed, indicating that exact wording was less accurately retained in the case of concrete than abstract sentences. This is analogous to the Begg and Paivio (1969) finding that lexical changes were more likely to be detected when the sentences were abstract than when they were concrete.
One possible alternative interpretation of the present findings involves a common code theory, eliminating the necessity for separate imaginal and verbal codes. For example, Franks and Bransford (1972) have suggested that individuals may integrate the meaning of abstract as well as concrete sentences into holistic representations of the complete idea. On the basis of this hypothesis, it might have been expected that the recognition ratings for the abstract same and synonym sentences would not differ significantly in the present study, since a holistic representation may have been generated for each of the abstract sentences in the initial comprehension rating task. The results, however, were inconsistent with this interpretation.

Generally, the problem faced by any common code interpretation of the present study is the simultaneous explanation of the nonsignificant difference between concrete-same vs concretesynonym ratings and the significant difference between abstract sentence ratings for the same two sentence-type conditions. Dual coding seems to have the advantage that it accurately predicts the general pattern of results for all conditions. This does not preclude the possibility that other tasks might require more complex theoretical interpretations, such as the assumption of a common (abstract) mode of representation in addition to imaginal and verbal codes (e.g., Marschark \& Paivio, in press; Paivio, 1976).

\section{REFERENCE NOTE}

1. Rowe, E. J., Schurr, B., \& Meisinger, D. Cued recall of concrete and abstract sentences. Unpublished manuscript. Memorial University, 1973.

\section{REFERENCES}

Begg, I., \& Paivio, A. Concreteness and imagery in sentence meaning. Journal of Verbal Learning and Verbal Behavior, $1969,8,821-827$.

Clark, H. H. The language-as-fixed-effect fallacy: A critique of language statistics in psychological research. Journal of Verbal Learning and Verbal Behavior, 1973, 12, 335-359. P. 347. (a)

Franks, J. J., \& Bransford, J. D. The acquisition of abstract ideas. Journal of Verbal Learning and Verbal Behavior, 1972, 11, 311-315.

Johnson, M. K., Bransford, J. D., Nyberg, S. E., \& Cleary, J. J. Comprehension factors in interpreting memory for abstract and concrete sentences. Journal of verbal Learning and Verbal Behavior, 1972, 11, 451-454.

MARSCHARK, M., \& PaIvio, A. Integrative processing of concrete and abstract sentences. Journal of Verbal Learning and Verbal Behavior, in press.

MoEser, S. D. Memory for meaning and wording in concrete and abstract sentences. Journal of Verbal Learning and Verbal Behavior, 1974, 13, 682-697.

PaIvio, A. The relationship between verbal and perceptual codes. In E. C. Carterette \& M. P. Friedman (Eds.), Handbook of perception (Vol. 9): Perceptual processing. New York: Academic Press, 1976.

Paivio, A., \& Begg, I. Imagery and comprehension latencies as a function of sentence concreteness and structure. Perception \& Psychophysics, 1971, 10, 408-412.

Paivio, A., Yuille, J. C., \& Madigan, S. A. Concreteness, imagery, and meaningfulness values for 925 nouns. Journal of Experimental Psychology, Monograph Supplement, 1968, 76, entire.

(Received for publication November 5, 1976.) 